\title{
Association Between Secondary Peritoneal Hydatidosis and Peritoneal Carcinomatosis from Ovarian Cancer - A Case Report
}

\author{
NICOLAE BACALBASA ${ }^{1 *}$, IRINA BALESCU ${ }^{2}$, IULIAN BREZEAN $^{1,3^{*}}$, \\ MIHAELA VILCU ${ }^{1,3^{*}}$ and VLADISLAV BRASOVEANU ${ }^{4}$ \\ 1 "Carol Davila" University of Medicine and Pharmacy, Bucharest, Romania; \\ 2 "Ponderas" Academic Hospital, Bucharest, Romania; \\ 3 “I. Cantacuzino” Clinical Hospital, Bucharest, Romania; \\ 4 "Dan Setlacec" Center of Gastrointestinal Disease and Liver Transplantation, \\ Fundeni Clinical Institute, Bucharest, Romania
}

\begin{abstract}
Peritoneal carcinomatosis from ovarian cancer is unfortunately a very common finding, especially in patients diagnosed in very late stages of the disease. Nonetheless, it is not the only pathological condition inducing a diffuse involvement of the peritoneum; other entities can have an infectious or miscellaneous origin. However, the association of peritoneal carcinomatosis and parasitic infection has never been reported so far. We present the case of a 50-year-old patient who had been submitted to surgery for a hepatic hydatid cyst. Four years later, the patient was diagnosed with disseminated peritoneal lesions, which proved to have both parasite and neoplastic origin. The patient was re-submitted to surgery, and debulking resection of all pathological lesions was achieved. Although it is very rare, the association of peritoneal carcinomatosis and peritoneal hydatidosis should be taken into consideration whenever the patient reports a suggestive medical history for those two pathological findings.
\end{abstract}

Peritoneal involvement can be frequently encountered in patients with various malignancies. However, the peritoneal disease can be induced by several pathological entities, including benign neoplastic lesions such as peritoneal leiomyomatosis, infectious entities such as peritoneal

This article is freely accessible online.

*The Authors contributed equally to this study.

Correspondence to: Irina Balescu, 2A Caraiman Street, 120143, Buzau, Romania. Tel: +40 724077709, e-mail: irina.balescu@ponderas-ah.ro

Key Words: Peritoneal carcinomatosis, peritoneal hydatidosis, debulking surgery. hydatidosis, tuberculosis, and mycobacterial peritonitis, as well as miscellaneous causes such as dropped gallstones (1).

Hydatid disease represents a zoonotic infectious disease caused by the presence of Echinococcus granulosus larvae, usually involving the liver (in up to $70 \%$ of cases) and the lung (in up to $35 \%$ of cases) (1). Other less common locations include spleen, bone, and kidney (2). Peritoneal hydatidosis is rare, reported in about $13 \%$ of patients diagnosed with abdominal parasitic infection, being rather secondary than primary hydatid disease (3). Secondary peritoneal involvement is usually developed after a spontaneous intraabdominal rupture of a hydatid cyst or after iatrogenic spread during surgery for primary hepatic hydatidosis (1).

\section{Case Report}

The 50-year-old female had previously been diagnosed with a fissured left lobe hepatic hydatid cyst, thus inactivation of the parasite followed by evacuation and partial pericystectomy was performed. Postoperative course was uneventful; hence, the patient was discharged in the sixth postoperative day and was confined to the infectious disease service in order to complete the therapeutic protocol by the administration of albendazole. The postoperative evaluations performed at six months follow-up showed no sign of recurrent hydatid disease. However, four years after the initial diagnosis, the patient complained of diffuse abdominal pain, weight loss, and asthenia. A computed tomography was then performed, demonstrating the presence of diffuse peritoneal calcifications along with peritoneal thickening and tumoral transformation of the two adnexa, with no demarcation line between the adnexal areas and the rectum (Figures 1 and 2). In the meantime the laboratory studies demonstrated an increased level of cancer antigen 125 (CA125) and antiEchinococcus antibodies. The patient was re-submitted to 


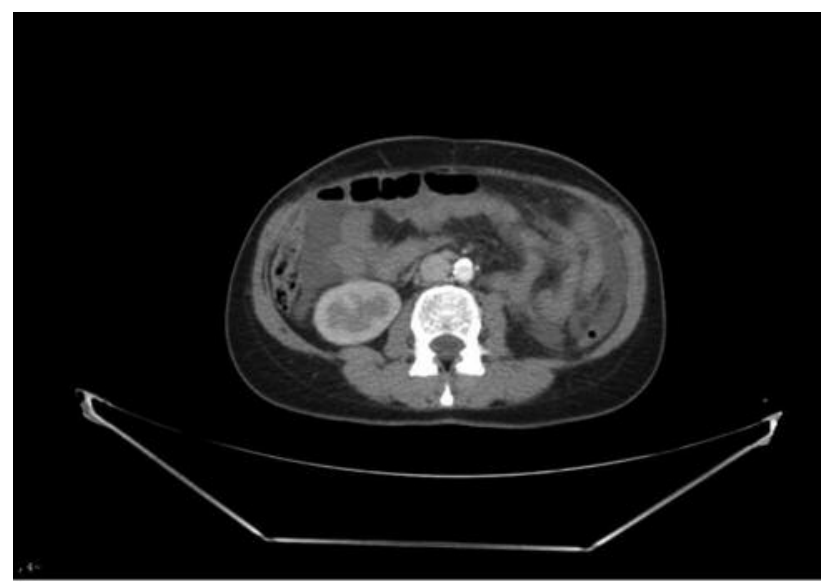

Figure 1. Diffuse peritoneal thickening.

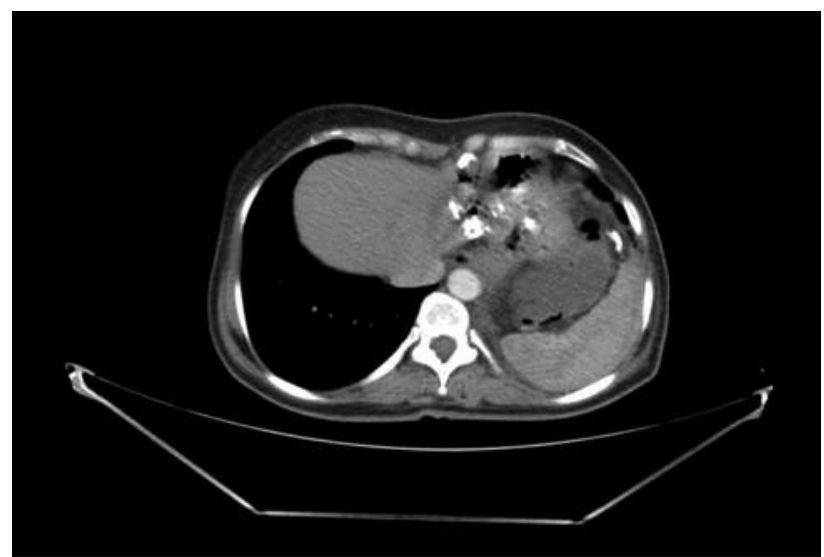

Figure 2. Peri-hepatic and intraperitoneal calcifications.

surgery, and the diagnosis of secondary peritoneal hydatidosis in association with peritoneal carcinomatosis with ovarian origin was confirmed intraoperatively. A total hysterectomy with bilateral adnexectomy and rectosigmodian resection along with total omentectomy, pelvic and para-aortic lymph node dissection, pelvic and parietal peritonectomy were performed. Complete excision of all hydatid lesions was achieved (Figures 3-7). The histopathological studies confirmed the presence of a bilateral high-grade serous ovarian cancer invading the uterine body, the rectosigmoidian loop, as well as the omentum. Moreover, focal invasion areas of the malignant process and hydatid lesions at the level of the peritoneum were reported. The histopathological studies also demonstrated the presence of tumoral vascular emboli, while no sign of perineural invasion was encountered; none of the 24 resected lymph nodes presented tumoral invasion.

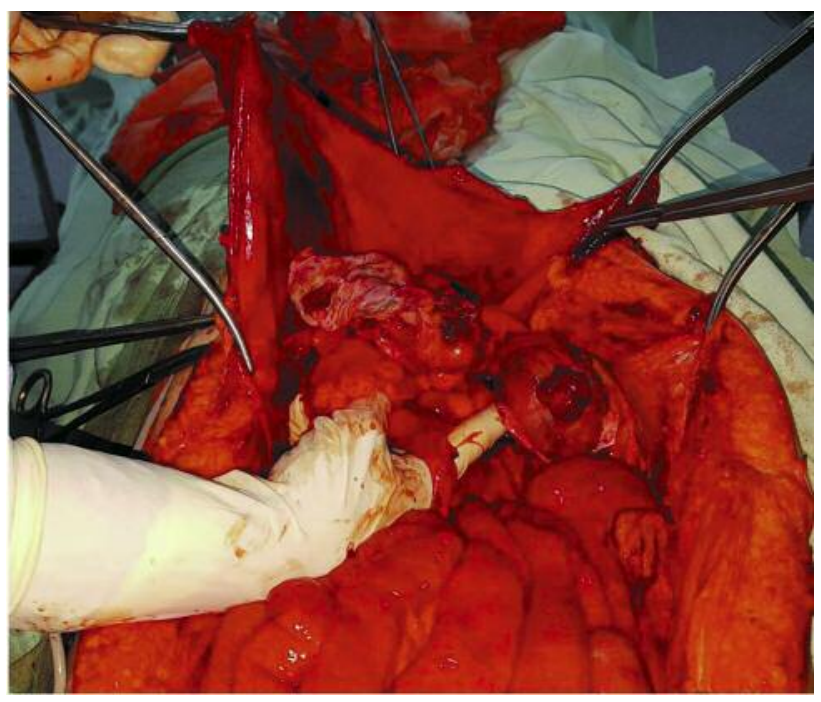

Figure 3. The aspect after mobilization of the parietal and pelvic peritoneum.

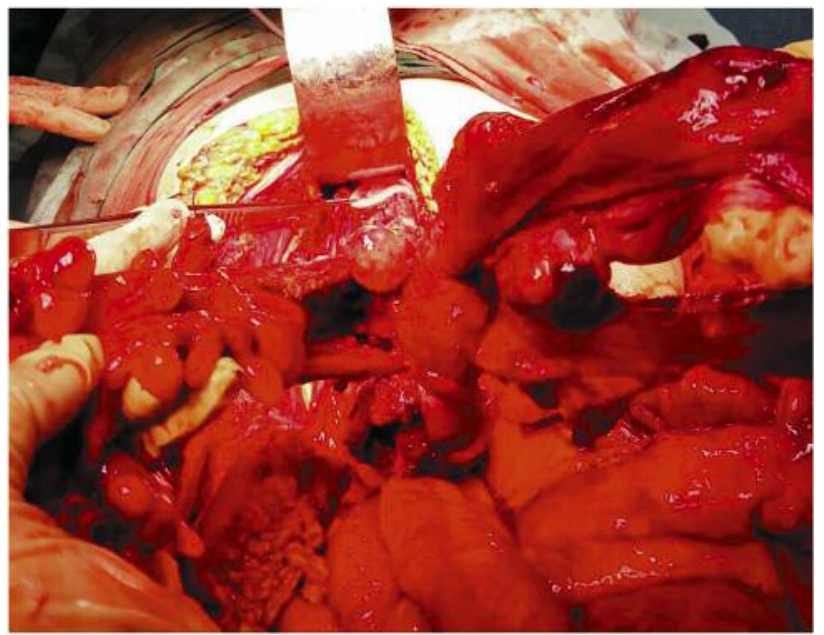

Figure 4. The aspect after mobilization of the rectosigmoidian loop en bloc with total hystrerectomy, bilateral adnexectomy, and extended peritoneal resections.

The postoperative course was uneventful, and the patient was confined to both oncology and infectious disease service at one month postoperatively.

\section{Discussion}

Although hepatic and pulmonary hydatidosis represent the most common localizations of this pathology, it seems that hydatidosis can develop at the level of any anatomic site $(4,5)$. The most common patterns of spread are the hematogenous 


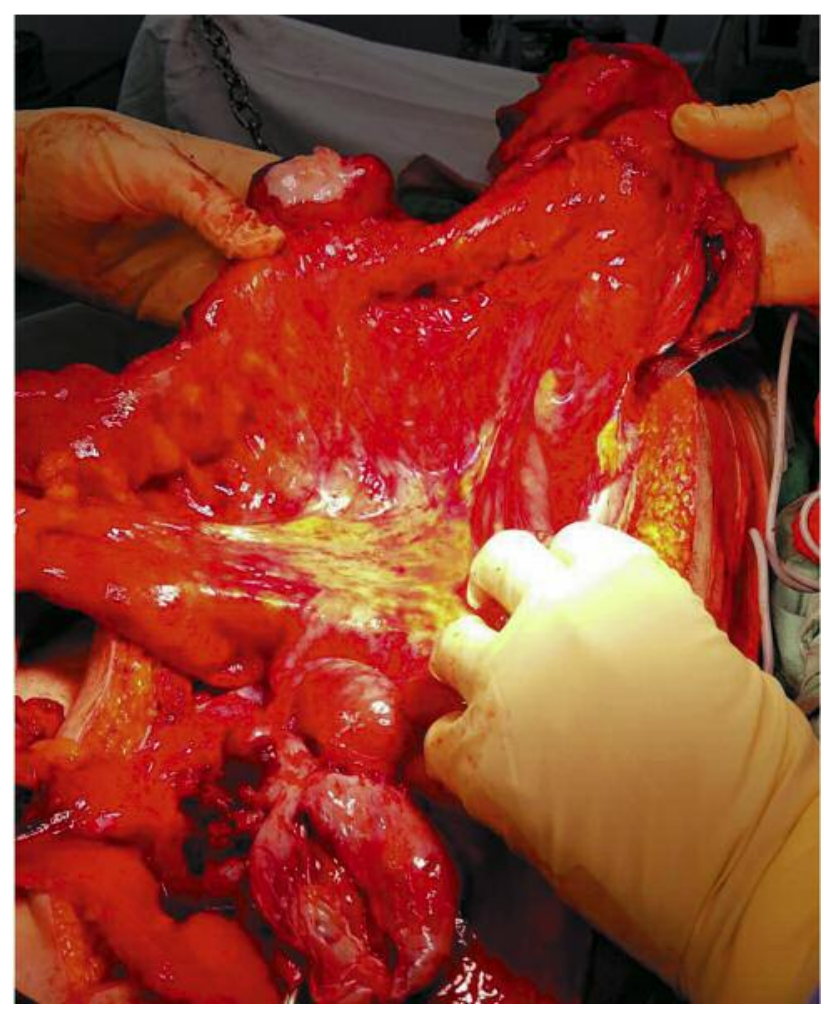

Figure 5. Hydatid lesion on the peritoneal surface of the rectosigmoidian loop.

route, which is most frequently responsible for the apparition of both intra- and extra-abdominal hydatid lesions, and peritoneal involvement, which accounts for up to $13 \%$ of all intra-abdominal hydatidosis $(4,5)$. The latter is classified into primary and secondary disease; primary lesions are rarely encountered, while secondary disease usually occurs after a rupture of a visceral intra-abdominal cyst. Once this phenomenon occurs, the parasite will migrate through the peritoneal route and will lead to the apparition of disseminated intraperitoneal lesions $(6,7)$.

The diagnosis of peritoneal hydatidosis is based on the medical history of the patient, as well as on the laboratory and imaging investigation. The most efficient imaging modality to identify this pathology is computed tomography $(8,9)$, and the presence of cyst wall calcifications, septae as well as the aspect of the internal content is the most common finding (4). In addition, computed tomography can be used in order to estimate the response of the patient to the neoadjuvant antihelminthic drugs (4).

However, differential diagnosis between peritoneal hydatidosis and peritoneal carcinomatosis can sometimes be difficult to be established preoperatively. In a case report in 2014, the final diagnosis of hydatidosis was established

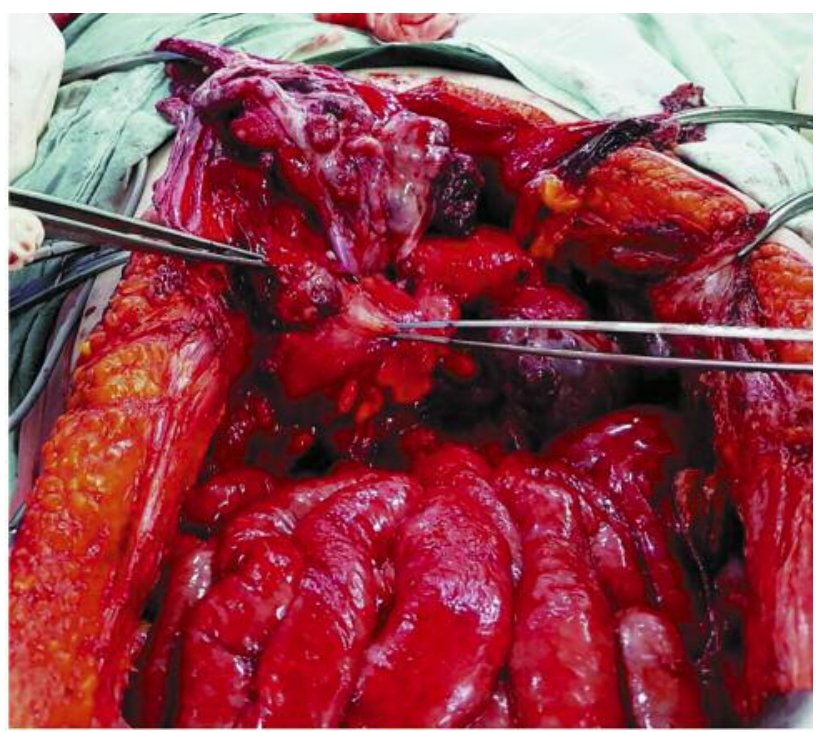

Figure 6. Mobilization of the pelvic mass, which, including the uterus, the rectosigmoidan loop and the peritoneum, presented lesions of carcinomatosis and hydatidosis.

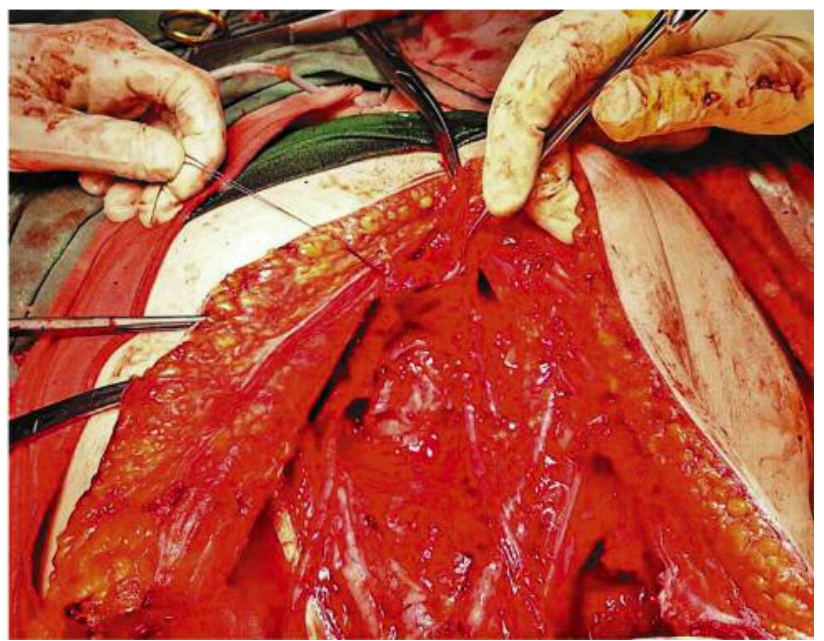

Figure 7. The final aspect after debulking surgery - total hysterectomy with bilateral adnexectomy en bloc with rectosigmoidian resection, pelvic and parietal peritonectomy, total omentectomy and pelvic lymph node dissection.

intraoperatively. The authors described the case of a 65 -year-old patient who presented with increased the abdominal diameter that had been observed in the last three months. The patient was diagnosed preoperatively with a $10 \times 6 \mathrm{~cm}$ cyst originating from one of the ovaries, while the serum levels of CA125 were normal. The patient was submitted to surgery and a large primary peritoneal hydatid case was diagnosed intraoperatively (10). 
Another similar case was described by Sing et al. in 2010; a 27-year-old patient was investigated for abdominal pain and menorrhagia and was diagnosed with a $11 \times 7 \times 3 \mathrm{~cm}$ cyst arising from the right adnexa, presenting multiple septae and solid areas. The preoperative levels of CA125 were slightly elevated (measuring 60-70 U/ml); thus, the patient was submitted to surgery. Intraoperatively, a right-sided cyst adherent to the right adnexa and to the mesentery was found, and was removed en bloc with total hysterectomy and bilateral adnexectomy. However, the histopathological findings confirmed the hydatid origin of the cyst (11).

An interesting study regarding extra-hepatic and extrapulmonary hydatidosis was published in 2017 by Adel et al (2). The study included 18 patients with extra-hepatic and extra-pulmonary hydatidosis diagnosed between May 2007 and December 2014 and submitted to surgery at the University Hospital of Guadalajara, Spain. Among these patients, two (83 and 46 years old) were diagnosed with disseminated peritoneal hydatidosis. Although the first patient had been submitted to surgery 30 years ago, at that moment, partial cystectomies for multiple intra-hepatic and peritoneal hydatidosis were performed. The second patient had initially been submitted to surgery 14 years ago for an intra-abdominal rupture of a hydatid cyst following an abdominal trauma, and then to other four further surgeries for recurrent intra-abdominal hydatidosis. At the moment of the study, the first patient refused any surgical procedure, while the second patient was submitted to an endoscopic retrograde cholangiopancreatography and prosthesis placement in order to interrupt the cysto-biliary communication. After a mean follow-up of 32 months, the two patients with disseminated peritoneal hydatidosis remained clinical asymptomatic. Among the 18 patients included in that study, only one was diagnosed with a synchronous malignant process. Specifically, a 73-year-old patient had a stage IV gallbladder cancer and a solitary splenic hydatid cyst. However, in this case no surgery was performed and the patient died of disease at the end of the study (2).

In patients diagnosed with disseminated peritoneal hydatidosis, the most common therapeutic options include periodic observation in association with albendazole treatment or percutaneous drainage (puncture, aspiration, injection, reaspiration), recommended especially in patients with disseminated lesions and high surgical risk.

Concerning surgery recommendation, it should not be omitted that another surgical procedure in patients diagnosed with peritoneal hydatidosis, who have undergone many previous laparotomies, is often associated with a higher morbidity rate. In consequence, surgery should be reserved only for patients presenting signs of complications such as debilitating pain or intestinal obstruction. Another special situation, in which surgery is imposed, is when the patient is diagnosed with other synchronous pathology that should be treated by surgery (2).
In our case, surgery was indicated by the presence of a synchronous ovarian carcinoma with peritoneal lesions. The main scope of the surgical procedure consisted in obtaining a complete cytoreduction in order to control the evolution of the neoplastic process. Moreover, the presence of the peritoneal lesions of hydatidosis in the absence of any visceral hydatid involvement was successfully resolved during the same surgical procedure, providing a significant improvement in the patient status.

The benefits of the cytoreductive surgery in patients with peritoneal lesions from ovarian cancer have been widely studied. The concept of cytoreductive surgery was first intuited by Meigs in 1934 (12). Four decades later, Griffiths demonstrated that a residual volume $<1.6 \mathrm{~cm}$ significantly improved the survival rate (13). Since that moment, attention was focused on studying whether an even smaller residual tumoral volume might improve survival. Therefore, other authors reported that a residual tumoral volume smaller than $1 \mathrm{~cm}$ or than $0.5 \mathrm{~cm}$ might induce a better outcome (14-16). This aspect was probably best demonstrated, in a metaanalysis by Bristow et al., which included 6,885 patients submitted to surgery for advanced stage ovarian cancer. The authors showed that each $10 \%$ increase of the proportion of patients submitted to maximal cytoreductive surgery induced a concomitant $5.5 \%$ increase of the median survival time of the entire cohort (17). Moreover, association of aggressive surgical procedures in both the lower and the upper abdomen induced a significant benefit in terms of survival without increasing the rate of postoperative complications (18-22). Therefore, multiple visceral resections have been successfully performed in order to achieve a maximal cytoreductive effort.

In the current case, the principles of debulking surgery were successfully applied inducing a complete cytoreduction of the tumoral masses with ovarian cancer origin. Moreover, satisfactory removal of the peritoneal lesions of hydatidosis was achieved, thus improving the immunological and clinical status of the patient who was further confined to the oncology department.

\section{Conclusion}

Although the most common pathological entity involving the peritoneal surface remains the neoplastic one, especially in patients with malignant diseases, other conditions such as infectious lesions should not be omitted especially in cases known with a previous history of hydatid disease. In such cases surgery is needed in order to achieve a good control of the malignant process; in the meantime excision of all parasitic lesions might be tempted in order to control both pathological processes. To the best of our knowledge, this is the first reported case of debulking surgery for advanced stage ovarian cancer in a patient with synchronous peritoneal 
hydatidosis. Moreover, performing an aggressive surgical approach addressed to both neoplastic and infectious process will improve the biological status of the patient, increasing the chances of association of an appropriate adjuvant oncological treatment.

\section{References}

1 Fasih N, Galwa RP, Macdonald DB, Fraser-Hill MA, McInnes $\mathrm{M}$ and Khalili K: Thinking beyond peritoneal carcinomatosis: imaging spectrum of unusual disseminated peritoneal entities. Can Assoc Radiol J 62: 125-134, 2011.

2 Adel F, Ramia JM, Gijon L, Plaza-Llamas R, Arteaga-Peralta V and Ramiro-Perez C: Extrahepatic and extrapulmonary hydatidosis. Cir Cir 85: 121-126, 2017.

3 Chawla A, Maheshwari M, Parmar H, Hira P and Hanchate V: Imaging features of disseminated peritoneal hydatidosis before and after medical treatment. Clin Radiol 58: 818-820, 2003.

4 Pedrosa I, Saiz A, Arrazola J, Ferreiros J and Pedrosa CS: Hydatid disease: radiologic and pathologic features and complications. Radiographics 20: 795-817, 2000.

5 Pantea S, Jiga L, Ionac M and Lazar F: Arterio-venous fistula using nonpenetrating titanium clips (VCS). Chirurgia (Bucur) 108: 563-567, 2013.

6 Ilica AT, Kocaoglu M, Zeybek N, Guven S, Adaletli I, Basgul A, Coban H, Bilici A and Bukte Y: Extrahepatic abdominal hydatid disease caused by Echinococcus granulosus: imaging findings. AJR Am J Roentgenol 189: 337-343, 2007.

7 Karavias DD, Vagianos CE, Kakkos SK, Panagopoulos CM and Androulakis JA: Peritoneal echinococcosis. World J Surg 20: 337-340, 1996.

8 Dar MA, Shah OJ, Wani NA, Khan FA and Shah P: Surgical management of splenic hydatidosis. Surg Today 32: 224-229, 2002.

9 Matheu V, Gracia MT, Rodríguez VM, Olalde S and Baeza ML: Shock anafiláctico secundario a rotura espontánea de quiste hidatídico esplénico. Rev Esp Alergol Inmunol Clín 12: 242-247, 1997.

10 Pagaro PM, Chaudhari PK, Naphade NN and Patil T: Isolated peritoneal hydatidosis clinically mimicking ovarian tumor: A rare case report. Med J DY Patil Univ 7(1): 78-81, 2014.

11 Sing P, Mushtaq D, Verma $\mathrm{N}$ and Mahajan NC: Pelvic hydatidosis mimicking a malignant multicystic ovarian tumor. Korean J Parasitol 48: 263-265, 2010.

12 Meigs JV: Tumors of the female pelvic organs. New York: Macmillan, 1934
13 Griffiths CT: Surgical resection of tumor bulk in the primary treatment of ovarian carcinoma. Natl Cancer Inst Monogr 42: 101-104, 1975

14 Hoskins WJ, Bundy BN, Thigpen JT and Omura GA: The influence of cytoreductive surgery on recurrence-free interval and survival in small-volume stage III epithelial ovarian cancer: a Gynecologic Oncology Group study. Gynecol Oncol 47: 159166, 1992.

15 Hoskins WJ, McGuire WP, Brady MF, Homesley HD, Creasman WT, Berman M, Ball H and Berek JS: The effect of diameter of largest residual disease on survival after primary cytoreductive surgery in patients with suboptimal residual epithelial ovarian carcinoma. Am J Obstet Gynecol 170: 974-979, 1994.

16 Bacalbasa N, Dima S, Balescu I, David L, Brasoveanu V and Popescu I: Results of primary cytoreductive surgery in advanced-stage epithelial ovarian cancer: A single-center experience. Anticancer Res 35: 4099-4104, 2015.

17 Bristow RE, Tomacruz RS, Armstrong DK, Trimble EL and Montz FJ: Survival effect of maximal cytoreductive surgery for advanced ovarian carcinoma during the platinum era: a metaanalysis. J Clin Oncol 20: 1248-1259, 2002.

18 Bacalbasa N, Balescu I, Dima S, Brasoveanu V and Popescu I: Pancreatic resection as part of cytoreductive surgery in advanced-stage and recurrent epithelial ovarian cancer--A singlecenter experience. Anticancer Res 35: 4125-4129, 2015.

19 Bacalbasa N, Dima S, Brasoveanu V, David L, Balescu I, Purnichescu-Purtan R and Popescu I: Liver resection for ovarian cancer liver metastases as part of cytoreductive surgery is safe and may bring survival benefit. World J Surg Oncol 13: 235, 2015.

20 Bacalbasa N, Balescu I, Dima S, Brasoveanu V and Popescu I: Splenectomy as part of cytoreductive surgery in recurrent epithelial ovarian cancer. Anticancer Res 35: 5097-5101, 2015.

21 Bacalbasa N, Balescu I, Dima S, Brasoveanu V and Popescu I: Hematogenous splenic metastases as an independent negative prognosis factor at the moment of primary cytoreduction in advanced stage epithelial ovarian cancer - A single center experience. Anticancer Res 35: 5649-5654, 2015.

22 Bacalbasa N, Balescu I and Dima S: Rectosigmoidian involvement in advanced-stage ovarian cancer - Intraoperative decisions. In Vivo 31: 973-977, 2017.

Received February 20, 2018

Revised April 2, 2018

Accepted April 5, 2018 